\title{
Psychological resilience associates with pain experience in women treated for breast cancer
}

\section{Liesto, Sanna}

2020-07

Liesto , S , Sipilä , R , Aho , T , Harno , H , Hietanen , M \& Kalso , E 2020 , ' Psychological resilience associates with pain experience in women treated for breast cancer ' , Scandinavian journal of pain , vol. 20 , no. 3 , pp. 545-553 . https://doi.org/10.1515/sjpain-2019-0137

http://hdl.handle.net/10138/329375

https://doi.org/10.1515/sjpain-2019-0137

acceptedVersion

Downloaded from Helda, University of Helsinki institutional repository.

This is an electronic reprint of the original article.

This reprint may differ from the original in pagination and typographic detail.

Please cite the original version. 
Psychological resilience associates with pain experience in women treated for breast cancer

S. Liesto ${ }^{1,2}$, MPs, PhD student, R. Sipilä ${ }^{1}$, MPs, PhD, T. Aho ${ }^{1}$ MPs, PhD student, H. Harno ${ }^{1,3}$, MD, PhD, M. Hietanen ${ }^{2}, \mathrm{MPs}, \mathrm{PhD}, \mathrm{E} . \mathrm{Kalso}^{1}, \mathrm{MD}, \mathrm{PhD}$

1) Division of Pain Medicine, Dept. of Anaesthesiology, Intensive Care and Pain Medicine, University of Helsinki and Helsinki University Hospital, Finland

2) Neurocenter, Neuropsychology, University of Helsinki and Helsinki University Hospital, Finland

3) Neurocenter, Neurology, University of Helsinki and Helsinki University Hospital, Finland

Running title: Pain, resilience and anxiety in breast cancer survivors

Key words: Anxiety, Cancer, Pain Severity, Pain Interference, Psychological Resilience, Relative Pain Interference

Corresponding author: Sanna Liesto, Helsinki University Hospital, Haartmaninkatu 2 A, PL 140, 00029 HUS, Finland. Phone +358 503795874 


\section{Abstract \\ Background and aims}

Psychological resilience refers to successful adaptation or a positive outcome in the context of significant life adversity, such as chronic pain. On the other hand, anxiety closely associates with pain. The aim of this study was to explore how anxiety and psychological resilience together associate with persistent and experimental pain.

\section{Methods}

In a cross-sectional design, we studied 160 patients who had previously been treated for breast cancer and who now reported at least moderate pain (NRS $\geq 4$ ) in any area of the body. Psychological resilience was measured on the Resilience Scale-14, anxiety on the Hospital Anxiety and Depression Scale, and intensity and interference of persistent pain by means of the Brief Pain Inventory. The cold pressor test was conducted to assess sensitivity to experimental cold pain.

\section{Results}

The results showed that resilience associated with pain interference in persistent pain, and that anxiety moderated this effect. Higher psychological resilience was associated with lower pain interference and this association was stronger in patients with low anxiety than among patients with high anxiety. These effects were visible with regard to persistent pain but not in experimental cold pain.

\section{Conclusions}

These results indicate that chronic pain and experimental pain as well as pain severity and pain interference are psychologically different phenomena. Psychological resilience protects against pain interference but effectively only in patients with low anxiety. It is necessary also to consider protective factors in addition to vulnerability factors in cases of persistent pain.

\section{Implications}

Resilience has been considered a potential target for intervention in chronic pain. However, high levels of anxiety might diminish the protective effect of psychological resilience in clinical settings. Therefore, it is important to treat anxiety in addition to resilience enhancing interventions. Patients with low psychological distress might be more suitable for resilience enhancing interventions than patients with high anxiety. 


\section{Introduction}

Breast cancer is the most common cancer among women in Western countries (1). Treatments have improved over the years, and hence more women survive and live with the possible consequences of cancer treatment in terms of their psychological wellbeing and health-related quality of life (HRQoL). Persistent pain follows treatment for breast cancer in 13-65 per cent of cases, depending on the assessment method $(2,3)$, and may lead to depression and disability in some whereas others remain unaffected (4). Pain is a distressing experience associated with actual or potential tissue damage with sensory, emotional, cognitive and social components (5). Pain experience can be conceptualized as two entities of pain severity and pain interference (6).

Psychological resilience means successful adaptation or a positive outcome in the context of significant adversity (7). It is an independent construct from the field of positive psychology that cannot be dismissed as the opposite of negative psychological factors or the absence of disability (8). Opinions vary on whether resilience is a stable characteristic, comparable to a personality trait, or a more modifiable and dynamic process $(7,9)$. Resilience as a construct, and the methods used to measure it, also vary between studies. Resilience may be measured as absence of psychiatric symptoms (e.g. depression), in terms of traits enhancing positive coping (e.g. high positive emotion), or directly on scales comparable to personality traits (9). This makes it difficult to compare the different studies on resilience and it may explain the varying results between them. In this study, resilience is defined as a group of characteristics that promotes adaptation in the face of adversity and which is measured with the Resilience Scale -14, RS-14.

Anxiety is known to associate with pain experience (10). Pain attracts more attention in anxious individuals (11) and is therefore a vulnerability factor in the context of pain. In the context of pain, resilience has recently been conceptualized as enabling the patient to recover from disability and depression, and to sustain functioning in the presence of pain (8). It therefore plays an important role in the process of coping and adjusting with chronic pain (12). Among patients with chronic pain, resilience has been suggested to have a positive association with quality of life (13), and a negative association with pain severity (14) and interference (15), disability (16) and depressive symptoms (17).

Persistent postsurgical pain (PPSP) in women treated for breast cancer has been actively studied (18). We have previously reported that breast cancer survivors with PPSP also report more other chronic pains than those who do not have PPSP (19). Therefore, we included in this study all areas of pain the participant reported, not only pain in the surgical area. In order to study pain interference independently from pain severity, "relative pain interference" has been used in previous studies (20). Experimental pain is often studied in connection with persistent pain to assess sensitization to pain $(19,21)$. Measures of pain 
sensitivity are predictive of acute postoperative pain and can be a risk for chronic pain conditions (22). Chronic pain and experimental pain are different in nature. In a study of experimental pain, the negative relationship between resilience and relative pain interference was stronger in individuals with high anxiety compared to individuals with low anxiety (20).

The aim of this study was to analyse the association between psychological resilience and pain in breastcancer survivors with persistent pain. We hypothesized that psychological resilience would associate negatively with pain severity and pain interference in the cases of both persistent and experimental pain. We also hypothesized that psychological resilience would associate with relative pain interference. Finally, we hypothesized that anxiety would moderate this effect so that the association is stronger among participants with high anxiety. We expected to see these effects both in experimental and in persistent pain.

\section{Methods}

Participants

The study was approved by the Coordinating Ethics Committee of the Helsinki and Uusimaa Hospital District (reference number 149/13/03/00/14). All participants gave their informed consent in writing. This is a cross-sectional study. The participants were recruited for the current NeuroPain study from the BrePainGen study, which assessed acute and persistent pain among 1,000 women treated for breast cancer at the Helsinki University Hospital during 2006-2010 (21). Detailed description of patient selection is presented in Figure 1. The participants with a surgical nerve lesion are described in more detail elsewhere (19).

The NeuroPain cohort consisted of 402 participants who filled in multiple questionnaires during the research visit which took place four to nine years after the initial breast-cancer surgery. Of these, 160 reported at least moderate (NRS $\geq 4$ ) pain severity or pain interference in any area of the body during last week and were included in the present study (Figure 1). Information about breast-cancer treatment was collected from the patients' records.

\section{Questionnaires}

Pain severity and interference

The Brief Pain Inventory (BPI) is a self-report measure that assesses two dimensions of pain: the intensity (severity) and the impact of pain on functioning (interference) (6). The inventory comprises four items measuring pain severity and seven items measuring pain interference during the past 24 hours on an 11 - 
point Likert scale (Numerical Rating Scale, NRS, $0=$ no pain, 10=the worst imaginable pain severity/very much interference). In this study, the 24 hour time frame was changed to "the past week" time frame. Given that this study is part of a project focusing on persistent postsurgical pain, the participants filled in the BPI separately for the surgical area (the breast, the upper arm or the axilla) and for other body areas in which they reported pain. The area in which the participant reported the highest NRS value for pain severity or pain interference during the past week was chosen as her pain report. If she reported the same highest NRS value for both surgical and other areas, the reports on the surgical area were used. The Pain Severity Score was calculated as the mean of the four severity items from the BPI. Similarly, the Pain Interference Score was calculated as the mean of the seven severity items from the BPI (6). Even though pain interference and pain intensity are independent constructs they are still correlated. Therefore, we calculated Relative Pain Interference to better capture pain interference independent from pain intensity. Relative Pain Interference was calculated by dividing the interference score by the severity score (20).

Psychological resilience

We used the short version of The Resilience Scale (RS-14) (23), translated into Finnish in an appropriate manner and validated in a Finnish healthy sample (24). This method constructs resilience as a group of characteristics representing resilience, which are perseverance, equanimity, meaningfulness, self-reliance, and existential aloneness (23). For each of the 14 items the respondents state the degree to which they agree or disagree on a seven-point Likert-type scale. Examples of the items are "I usually manage one way or another", "I am determined", and "My life has meaning". The total possible sum score ranges from 14 to 98 points, a higher score representing higher resilience. Cronbach's alpha in this study sample is 0.92 . Resilience was used as a continuous variable in the analyses.

Depressive symptoms and anxiety

The Hospital Anxiety and Depression Scale (HADS) is a 14-item questionnaire (25). Participant rate the items on a four-point Likert scale. The total sum score for both the depression and the anxiety subscales varies from zero to 21 . The clinically significant level of both depressive symptoms and anxiety is $\geq 8$ points on HADS-D and HADS-A, respectively (25). In these analyses we only used anxiety, which has been shown to have a stronger association with pain, and to avoid multicollinearity between depression and anxiety $(2,21)$.

\section{The Cold Pressor test}

The Cold Pressor test (CPT) was used to assess experimental pain sensitivity and tolerance. The participants immersed their hand in a bath with circulating cold water (+2-4 C) (JULABO USA Inc, Allentown, PA), holding it in up to the wrist for the maximum time tolerated or for up to 90 seconds. They reported pain severity every 15 seconds, and at the end of the CPT when they withdrew their hand from the water on an NRS 
ranging from zero to 10 . After the test, the participants also evaluated its unpleasantness on an NRS scale ranging from zero to 10 . Relative pain unpleasantness was calculated by dividing the unpleasantness score by the pain-intensity score at the end of the test (20).

Statistical analysis

In cases of single missing items on the questionnaires (less than 20 per cent missing values on one questionnaire), the missing values were replaced with the means of the participant's responses from the same questionnaire. If the missing values amounted to more than 20 per cent of the questionnaire it was removed from the analyses. When reporting descriptive statistics (Table 1), we report means and standard deviations when the variables are normally distributed and medians and inter quartile ranges when the variables are skewed. Pearson's correlation was used to describe the strength of the association between resilience and pain severity or interference. Relative pain interference was calculated by dividing the paininterference score by the pain-intensity score. Since linear regression is sensitive to the effect of outliers, we removed one participant with an dependent variable 6.4 standard deviations above the mean. We used the enter method in linear regression and created two models. In the first model we analyzed the association between resilience and relative pain interference. In the second model we entered resilience, anxiety and the interaction term (resilience $x$ anxiety). The same analysis was conducted for resilience, anxiety and relative pain unpleasantness in the CPT. Resilience and anxiety were used as continuous variables. Anxiety was grouped into high and low groups for illustrative purposes. SPSS 22.0 version for Windows (SPSS INC, Chicago, IL) was used for the statistical analyses.

\section{Results}

We present the results for resilience and pain and the moderating effect of anxiety first for clinical pain and then for experimental pain.

Table 1 provides information about the participants. Most of them (82\%) reported higher pain severity or pain interference in other than the surgical area. The typical body areas where the patients reported pain were joints $(n=114)$, back $(n=70)$, neck and shoulder $(n=50)$, head $(n=24)$. Eight participants answered the questions about pain in the surgical area but not those regarding possible other areas. In addition, on two questionnaires, one patient in each had missing values and were removed from the analysis. One patient had $9 \%$ of items missing on a third questionnaire. Associations between variables are presented in a correlation table (Table 2). Resilience associated negatively with anxiety and relative pain interference. Anxiety associated with multiple pain measures (Table 2).

Persistent Pain 
The association between resilience and pain severity was not significant (Figure 2a). However, pain interference and resilience had a significant negative correlation (Figure $\mathbf{2 b}$ ).

We created a linear regression model to predict relative pain interference by resilience, anxiety, and their interaction term as independent variables. In model 1, the resilience score was negatively associated with relative pain interference (Table 3 ). In model 2 , the resilience score was negatively associated, the anxiety score was positively associated, and there was also a significant interaction between resilience and anxiety on relative pain interference (Table 3). The interaction is visualized in Figure 3. The negative relationship between resilience and relative pain interference was stronger among participants with low anxiety than among those with high anxiety. Of the former, those with high resilience reported lower relative pain interference than those with low resilience. This relationship between relative pain interference and resilience was not as pronounced in participants with high anxiety.

Experimental pain

All 160 participants participated in the CPT and of them, 67 participants $(42 \%)$ held their hand in the icecold water in the CPT for the maximum of 90 seconds. There was no difference in the level of resilience between the participants who withdrew their hand before the maximum time and those who held it there for the maximum time (78.58 [12.60] vs. 76.29 [13.19], $t=-1.11, p=0.27)$. Neither the final NRS in the CPT nor unpleasantness at the end associated with psychological resilience $(r=-0.01, p=0.94 ; r=-0.06, p=$ 0.46 , respectively). The relative Pain Unpleasantness of the CPT was not associated with resilience $(\beta=-$ $0.03, p=0.754)$ or anxiety $(\beta=-0.006, p=0.955)$ in the linear regression model (enter method), and there was no interaction between them $(\beta=-0.065, p=0.500)$.

\section{Discussion}

The main finding of this study is that psychological resilience associates with relative pain interference and that anxiety moderates this effect among women treated for breast cancer. On the other hand, the direction of the moderating effect was to the opposite direction than what we hypothesised. The protective effect of psychological resilience on relative pain interference was less pronounced in participants with high anxiety. In accordance with our hypothesis, there was a negative association between pain interference and resilience, but no association between psychological resilience and pain severity. Resilience showed a protective effect and anxiety a moderating effect in clinical, but not in experimental pain.

Resilience among cancer survivors 
Women treated for breast cancer react differently to potentially traumatising events related to diagnosis and treatment. Some of them have negative psychological outcomes, whereas others show remarkable resilience and even post-traumatic growth (26). In the present study, the level of resilience was similar to previous reports of Finnish healthy sample (17-92 years) (24). Features like optimism, hope, and a sense of coherence and positive personality-related variables e.g. personal control and low neuroticism and social support are associated with higher psychological resilience among cancer patients (26). In some cases, experiencing and surviving cancer is a transformative process that fosters the capacity to enjoy small daily pleasures and to attach less importance to former areas of concern, which in turn is reflected in increased resilience (27).

\section{Resilience and pain}

It has been suggested that vulnerability and protective factors may work simultaneously when an individual adapts to chronic pain (28), but it is only recently that these factors have been studied simultaneously. Previous studies have suggested that anxiety may moderate the effect of resilience in cases of chronic and experimental pain $(20,29)$. We did find a moderating effect of anxiety on resilience and relative pain interference in those who had persistent pain: the effect of resilience was more pronounced in those with low anxiety. This could be attributable to the nature of resilience which can only enhance adjustment among people with a substantial level of psychological wellbeing. However, another study on experimental pain reported that the effect of resilience was more pronounced among people with high anxiety (20). It is possible that there are multiple other confounding factors. In a previous study, resilience was found to associate with pain-related dysfunction via specific pain beliefs (pain self-efficacy, pain catastrophizing and challenge appraisals) (30). The prevalence of anxiety symptoms in women treated for breast cancer varies between 17.9-33.3\%, which is higher than that in the average female population (31). Anxiety is an important vulnerability factor in chronic pain. Also, anxiety, in comparison with depressive symptoms, has been reported to associate more strongly with persistent pain (2).

We included participants reporting their worst pain NRS $\geq 4 / 10$ in our analysis ( $0=$ no pain, $10=$ worst imaginable pain). The NRS cut-off point $\geq 4 / 10$ has been widely used to identify patients with moderate-tosevere or clinically significant pain (32). Unlike in previous studies $(14,16)$, there was no direct association between psychological resilience and the pain-severity rating. On the other hand, we did find an association between resilience and pain interference, which is in line with a study on upper limb loss (15). The differences in the findings could be attributable to the different patient cohorts and the type of pain. Ankawi et al. (2017) studied a sample of people reporting ongoing pain on at least three days a week for six months or more (16), whereas Ruiz-Parraga et al. (2012) focused on patients with chronic musculoskeletal pain appearing on five or more days a week for at least six months (14). A pain-specific resilience scale was 
used in both studies, whereas we used a more general resilience scale. According to Ruiz-Parraga et al., however, the weak although statistically significant association between resilience and pain severity implies that resilience in chronic pain has a stronger relationship with low interference in daily functioning and low emotional distress than with the intensity of pain (14). Ankawi et al. also suggest that the relationship between resilience and intensity could be mediated by the acceptance of chronic pain and pain-related self-efficacy (16). After all, even though we found an association between resilience and pain interference in this study, the size of the effect is rather small. However, this is an important piece of information as it will help us better understand the complex phenomena of living with persistent pain. In this study, we created a theoretical model where state anxiety moderates the effect of resilience on relative pain interference. It would also be possible to create a model where resilience moderates the effect of anxiety on relative pain interference as the moderating effect is likely to be in both directions.

Experimental pain

In a previous study in an experimental pain setting, relative pain unpleasantness was associated with psychological resilience, and anxiety moderated this effect (20). We did not find such an association in experimental pain, which may be due to the different experimental tests applied: Hemington et al. (2017) used suprathreshold heat pain, whereas we used the cold pressor test. It is possible that anxiety and resilience affect pain severity differently in the different modalities. Additionally, Hemington et al. (2017) studied trait anxiety, whereas we studied state anxiety. In this study, we found a protective effect of resilience and a moderating effect of anxiety on relative pain interference in clinical pain but not on relative unpleasantness in experimental pain. Experimental pain is very different from clinical pain on the psychological level in that it does not represent a threat to the person. In fact, mild anxiety has been found to improve tolerance of heat pain in healthy subjects (33).

Study limitations and strengths

Our study had only female participants. Females have reported more health-related problems than men in earlier studies, and gender differences become more pronounced with increasing age (34). The results of previous studies on gender differences in psychological resilience are mixed $(13,24,35-38)$. It has been suggested that resilience operates differently in older than in younger adults $(39,40)$. At the time of the breast cancer treatments, the mean age of the present sample was $55.3(7.9)$ years which is slightly younger than that of the average age (60 years) of the breast cancer patients in Finland (Finnish Cancer Registry). In this current study, the age limit was set to 75 which explains why the study group had more younger patients. This limitation is unlikely clinically significant.

Another limitation is that this study has the cross sectional design. The results are therefore correlational only. Further limitation of the study is that the pain variables are skewed to the right. That; it also affects 
the relative pain interference variable that, which is created from the pain severity and pain interference variables since there is not much variance in these variables. However, the relative pain interference variable itself is normally distributed. A strength of the study is the minimal amount of missing values. Another strength of this study was that in addition we asked the participant to rate possible pain in the postsurgical area and in other areas separately, which provided a better picture of their overall pain problem.

\section{Clinical implications}

According to our results, anxiety moderated the effect of resilience on pain interference. However, it seems that persons with high anxiety cannot exploit the protective role of resilience. Therefore, it may be necessary in clinical settings to target the treatment of chronic pain differently according to the patient's level of anxiety. Appropriate treatment for anxiety may have to precede resilience-enhancing interventions. On the other hand, improving resilience among women treated for breast-cancer at an early stage could be beneficial in terms of inhibiting the development of high levels of anxiety.

Previous studies have suggested that resilience might be a viable target for intervention (41). Improving patients' resilience and resilient behaviour could be a significant step in the development of new treatments or rehabilitation for patients with persistent pain and for cancer survivors. The available evidence indicates a modest but consistent benefit of resilience-training programmes in improving a number of mental-health outcomes (42). There are proposed guidelines for research on resilience intervention (9), and more studies are needed on interventions targeted specifically at enhancing resilience among cancer survivors and patients with chronic pain.

Psychosocial vulnerability has long been an important factor in pain research, whereas protective factors, especially psychological resilience, have been included only in recent years. Vulnerability and protective factors work independently to some extent $(28,43)$, and both should therefore be included in studies on adjustment to chronic pain. Resilient functioning (assessing both recovery and sustainability) is of interest and should be addressed in future research (8). A pain-specific resilience scale has recently been introduced in studies on experimental and chronic pain $(16,29)$. It should also be tested on cancer survivors suffering from persistent pain.

Table 1. Descriptive statistics ( $\mathrm{n}=159)$ shown as Mean (Standard Deviation). 
Table 2. Correlation table.

Table 3. Regression analysis predicting relative pain interference.

Figure 1. Flow-chart of the patient selection from the original 1000 patient cohort.

Figure 2. a) Scatterplot of resilience score and severity of persistent pain. b) Scatterplot of resilience and interference of persistent pain.

Figure 3. Association of psychological resilience, anxiety and their interaction on Relative Pain Interference of persistent pain.

\section{Authors' statements:}

Research funding: This study was supported by a grant from the European Union Seventh Framework Program (FP7) under grant agreement no. 602891.

Conflicts of interest: Authors state no conflict of interest.

Informed consent: Informed consent has been obtained from all individuals included in this study.

Ethical approval: The study was approved by the Coordinating Ethics Committee of the Helsinki and Uusimaa Hospital District (reference number 149/13/03/00/14).

\section{References}

(1) GBD 2016 Disease and Injury Incidence and Prevalence Collaborators. Global, regional, and national incidence, prevalence, and years lived with disability for 328 diseases and injuries for 195 countries, 19902016: a systematic analysis for the Global Burden of Disease Study 2016. Lancet 2017 Sep 16;390(10100):1211-1259.

(2) Meretoja T, Leidenius M, Tasmuth T, Sipilä R, Kalso E. Pain at 12 Months After Surgery for Breast Cancer. Jama-Journal Of The American Medical Association 2014;311(1):90-Journal.

(3) Andersen KG, Kehlet H. Persistent pain after breast cancer treatment: a critical review of risk factors and strategies for prevention. J Pain 2011 Jul;12(7):725-746.

(4) Deshields T, Tibbs T, Fan MY, Taylor M. Differences in patterns of depression after treatment for breast cancer. Psychooncology 2006 May;15(5):398-406.

(5) Williams AC, Craig KD. Updating the definition of pain. Pain 2016 Nov;157(11):2420-2423. 
(6) Cleeland CS, Ryan KM. Pain assessment: global use of the Brief Pain Inventory. Ann Acad Med Singapore 1994 Mar;23(2):129-138.

(7) Luthar SS. The Construct of Resilience: A Critical Evaluation and Guidelines for Future Work. Child Dev 2000;71(3):543.

(8) Goubert L, Trompetter H. Towards a science and practice of resilience in the face of pain. European Journal of Pain 2017.

(9) Chmitorz A, Kunzler A, Helmreich I, Tuscher O, Kalisch R, Kubiak T, Wessa M, Lieb K. Intervention studies to foster resilience - A systematic review and proposal for a resilience framework in future intervention studies. Clin Psychol Rev 2018 Feb;59:78-100.

(10) Keogh E, Cochrane M. Anxiety sensitivity, cognitive biases, and the experience of pain. J Pain 2002 Aug;3(4):320-329.

(11) Eccleston C, Crombez G. Pain demands attention: a cognitive-affective model of the interruptive function of pain. Psychol Bull 1999 May;125(3):356-366.

(12) Wright $L$, Zautra AJ, Going S. Adaptation to early knee osteoarthritis: the role of risk, resilience, and disease severity on pain and physical functioning. Ann Behav Med 2008 Aug;36(1):70-80.

(13) Battalio SL, Silverman AM, Ehde DM, Amtmann D, Edwards KA, Jensen MP. Resilience and Function in Adults With Physical Disabilities: An Observational Study. Arch Phys Med Rehabil 2016 Dec 18.

(14) Ruiz-Parraga GT, Lopez-Martinez AE, Gomez-Perez L. Factor structure and psychometric properties of the resilience scale in a spanish chronic musculoskeletal pain sample. J Pain 2012 Nov;13(11):1090-1098.

(15) Walsh MV, Armstrong TW, Poritz J, Elliott TR, Jackson WT, Ryan T. Resilience, Pain Interference, and Upper Limb Loss: Testing the Mediating Effects of Positive Emotion and Activity Restriction on Distress. Arch Phys Med Rehabil 2016 May;97(5):781-787.

(16) Ankawi B, Slepian PM, Himawan LK, France CR. Validation of the Pain Resilience Scale in a Chronic Pain Sample. The Journal of Pain 2017.

(17) Bauer H, Emeny R, Baumert J, Ladwig K. Resilience moderates the association between chronic pain and depressive symptoms in the elderly. European journal of pain 2016;20(8):1253-1265.

(18) Tait RC, Zoberi K, Ferguson M, Levenhagen K, Luebbert RA, Rowland K, Salsich GB, Herndon C. Persistent Post-Mastectomy Pain: Risk Factors and Current Approaches to Treatment. J Pain 2018 Dec;19(12):1367-1383.

(19) Mustonen L, Aho T, Harno H, Sipila R, Meretoja T, Kalso E. What makes surgical nerve injury painful? A 4-year to 9-year follow-up of patients with intercostobrachial nerve resection in women treated for breast cancer. Pain 2018 Oct 30.

(20) Hemington KS, Cheng JC, Bosma RL, Rogachov A, Kim JA, Davis KD. Beyond negative pain-related psychological factors: Resilience is related to lower pain affect in healthy adults. The Journal of Pain 2017.

(21) Kaunisto MA, Jokela R, Tallgren M, Kambur O, Tikkanen E, Tasmuth T, Sipila R, Palotie A, Estlander AM, Leidenius M, Ripatti S, Kalso E. Pain in 1,000 women treated for breast cancer: a prospective study of pain sensitivity and postoperative pain. Anesthesiology 2013 Dec;119(6):1410-1421.

(22) Nielsen CS, Staud R, Price DD. Individual differences in pain sensitivity: measurement, causation, and consequences. J Pain 2009 Mar;10(3):231-237.

(23) Wagnild GM, Young HM. Development and psychometric evaluation of the Resilience Scale. J Nurs Meas 1993 Winter;1(2):165-178. 
(24) Losoi H, Turunen S, Wäljas M, Helminen M, Öhman J, Julkunen J, Rosti-Otajärvi E. Psychometric Properties of the Finnish Version of the Resilience Scale and its Short Version. Psychology, Community \& Health 2013;2(1):1-10.

(25) Bjelland I, Dahl AA, Haug TT, Neckelmann D. The validity of the Hospital Anxiety and Depression Scale. An updated literature review. J Psychosom Res 2002 Feb;52(2):69-77.

(26) Seiler A, Jenewein J. Resilience in Cancer Patients. Front Psychiatry 2019 Apr 5;10:208.

(27) Padilla-Ruiz M, Ruiz-Roman C, Perez-Ruiz E, Rueda A, Redondo M, Rivas-Ruiz F. Clinical and sociodemographic factors that may influence the resilience of women surviving breast cancer: crosssectional study. Support Care Cancer 2019 Jan 4.

(28) Smith BW, Zautra AJ. Vulnerability and resilience in women with arthritis: test of a two-factor model. J Consult Clin Psychol 2008;76(5):799.

(29) Ruiz-Parraga G. T, Lopez-Martinez A. E, Esteve R, Ramirez_Maestre C, Wagnild G. A confirmatory factor analysis of the Resilience Scale adapted to chronic pain (RS-18): new empirical evidence of the protective role of resilience on pain adjustment. Quality Of Life Research 2015;24(5):1245-1253.

(30) Chen S, Jackson T. Pain beliefs mediate relations between general resilience and dysfunction from chronic back pain. Rehabil Psychol 2018 Nov;63(4):604-611.

(31) Maass SW, Roorda C, Berendsen AJ, Verhaak PF, de Bock GH. The prevalence of long-term symptoms of depression and anxiety after breast cancer treatment: A systematic review. Maturitas 2015 Sep;82(1):100-108.

(32) Gerbershagen HJ, Rothaug J, Kalkman CJ, Meissner W. Determination of moderate-to-severe postoperative pain on the numeric rating scale: a cut-off point analysis applying four different methods. $\mathrm{Br} \mathrm{J}$ Anaesth 2011 Oct;107(4):619-626.

(33) Starr CJ, Houle TT, Coghill RC. Psychological and sensory predictors of experimental thermal pain: a multifactorial model. J Pain 2010 Dec;11(12):1394-1402.

(34) Janssen MF, Szende A, Cabases J, Ramos-Goni JM, Vilagut G, Konig HH. Population norms for the EQ5D-3L: a cross-country analysis of population surveys for 20 countries. Eur J Health Econ 2018 Feb 14.

(35) Masood A, Masud Y, Mazahir S. Gender differences in resilience and psychological distress of patients with burns. Burns 2016 Mar;42(2):300-306.

(36) Terrill AL, Molton IR, Ehde DM, Amtmann D, Bombardier CH, Smith AE, Jensen M. Resilience, age, and perceived symptoms in persons with long-term physical disabilities. Journal of Health Psychology 2016 05/01; 2017/04;21(5):640-649.

(37) Ramirez-Maestre C, Esteve R. The role of sex/gender in the experience of pain: resilience, fear, and acceptance as central variables in the adjustment of men and women with chronic pain. J Pain 2014 Jun;15(6):608-618.e1.

(38) Bonanno GA, Galea S, Bucciarelli A, Vlahov D. What predicts psychological resilience after disaster? The role of demographics, resources, and life stress. J Consult Clin Psychol 2007 Oct;75(5):671-682.

(39) Hanfstingl B. Ego and spiritual transcendence: relevance to psychological resilience and the role of age. Evid Based Complement Alternat Med 2013;2013:949838.

(40) Duggleby W, Hicks D, Nekolaichuk C, Holtslander L, Williams A, Chambers T, Eby J. Hope, older adults, and chronic illness: a metasynthesis of qualitative research. J Adv Nurs 2012;68(6):1211-1223.

(41) Edwards KA, Alschuler KA, Ehde DM, Battalio SL, Jensen MP. Changes in Resilience Predict Function in Adults With Physical Disabilities: A Longitudinal Study. Arch Phys Med Rehabil 2017 Feb;98(2):329-336. 
(42) Leppin AL, Bora PR, Tilburt JC, Gionfriddo MR, Zeballos-Palacios C, Dulohery MM, Megan M, Sood A, Erwin PJ, Brito JP, Boehmer KR. The efficacy of resiliency training programs: a systematic review and metaanalysis of randomized trials. PLoS One 2014;9(10):e111420.

(43) Sturgeon JA, Zautra AJ, Arewasikporn A. A multilevel structural equation modeling analysis of vulnerabilities and resilience resources influencing affective adaptation to chronic pain. Pain 2014 Feb;155(2):292-298. 